\title{
Contenidos de isómeros trans de los ácidos grasos en productos cárnicos. (II) Tejido adiposo y grasa intramuscular del cerdo
}

\author{
Por M. C. Lluch, J. Pascual, J. Parcerisa, F. Guardiola, R. Codony, M. Rafecas y J. Boatella \\ Depto. de Ciencias Fisiológicas Humanas y de la Nutrición. Unidad de Nutrición y Bromatología. \\ Universidad de Barcelona.
}

\section{RESUMEN}

Contenidos de isómeros trans de los ácidos grasos en productos cárnicos. (II) Tejido adiposo y grasa intramuscular del cerdo.

Se presentan los resultados obtenidos en la determinación de ácidos grasos trans en una serie de muestras de tejidos subcutáneo y muscular, procedentes de canales de cerdo, mediante cromatografía en fase gaseosa. Ambos tipos de grasas presentan valores similares y relativamente bajos, con un valor medio del $0.6 \%$ de $\mathrm{C} 18: 1 \mathrm{t}$ Se detecta una marcada influencia de la alimentación, puesta de manifiesto por las diferencias significativas obtenidas para los contenidos de C18:1 trans, en función del origen de las muestras. Igualmente, se recogen las correlaciones obtenidas entre los contenidos de C18:1 t. y los de ácidos grasos saturados, monoinsaturados y poliinsaturados, así como con el contenido graso intramuscular.

PALABRAS-CLAVE: Acido graso - Cerdo - Grasa intramuscular Isómero trans (contenido) - Tejido adiposo.

\section{SUMMARY}

Trans isomers contents of the fatty acids in meat products. (II) Adipose tissue and intramuscular fat from pork.

We give the results obtained for the determination of trans fatty acids in subcutaneous and intramuscular fats from different pork samples, using gas chromatography. Values obtained are low and similar in both kind of fats, with a mean value of $0.6 \%$ of $C 18: 1$ trans. A clear influence of animal feeding is detected due to the significant differences of this isomer content between samples from different origin. Also, correlations have been observed between C18:1 t. content and saturated, mono, polinsaturated fatty acids and fat intramuscular contents.

KEY-WORDS: Adipose tissue - Fatty acid - Intramuscular fat - Pig Trans isomer (content).

\section{INTRODUCCION}

En un trabajo anterior (Hernández et al., 1991), se publicaron los datos obtenidos en el estudio de contenido de isómeros trans en productos cárnicos, con resultados parciales de un proyecto encaminado a establecer los niveles de dichas formas en alimentos y evaluar la ingesta de los mismos en nuestro modelo de consumo. En esta ocasión, se presentan los resultados obtenidos en el estudio de la fracción grasa intramuscular y en la de depósito subcutánea de canales de cerdo. En relación con ello, cabe destacar que no existen en la bibliografía datos relacionados con estos contenidos, correspondientes a animales criados en nuestro país.

La bibliografía relativa al contenido de estos isómeros en estos productos es escasa (Slover et al., 1987) (Lin et al., 1984) (Sommerfeld, 1983), y en ella se recogen cifras poco homogéneas. Esta variabilidad es lógica debido a que en el cerdo, como animal monogástrico, el contenido en ácidos grasos trans depende exclusivamente de la cantidad de los mismos presente en su alimentación (Sommerfeld, 1983). Este hecho diferencia claramente a las grasas con este origen de aquellas procedentes de bovino y ovino, que presentan cantidades más elevadas de estos ácidos como consecuencia de la formación adicional en el rúmen de dichos animales (Entressangles, 1986) (Brisson, 1982) (Gunstone y Norris, 1983).

La distribución de los distintos tipos de ácidos grasos a nivel de diferentes tejidos del cerdo, e incluso a nivel subcelular, es variable (Kinney et al., 1990) (Girard et al., 1987). Como demuestra Girard (1983) en estudios experimentales, las grasas de depósito en el cerdo son más susceptibles de modificar su composición que la grasa intramuscular. Por esta razón, cabría suponer que la incorporación de formas trans será también más elevada en tejidos grasos, a pesar de su menor contenido en ácidos grasos insaturados (Lin et al., 1984).

El interés nutricional de la presencia de ácidos grasos trans en la carne y productos derivados es relevante, dados sus posibles efectos biológicos no deseables, en el caso de ingestas elevadas (Zevenbergen, 1987) (Kummerow, 1986) (Emken, 1979) (British Nutrition Foundation-BNF, 1987) (Islam et al., 1983) (Kinsella et al., 1981). Por otra parte, los datos de consumo disponibles señalan una participación importante de las carnes y derivados en la ingesta total de ácidos trans para distintas poblaciones. Según la BNF (1987), en el Reino Unido esta contribución puede evaluarse en $1 \mathrm{~g} /$ persona/día, lo que supone un $15 \%$ de la ingesta total de ácidos trans. En los EE UU, Hunter et al. (1986) evalúan la participación de leche, carne y derivados en esta ingesta total en un $20 \%(1,4 \mathrm{~g} /$ persona/día). En España, los datos obtenidos por nosotros (Boatella, 1992) revelan una contribución del $29 \%$ ( $0.7 \mathrm{~g} /$ persona/día) correspondiente a la carne de bovino y del $4 \%$ ( $0.1 \mathrm{~g} /$ persona/día) para el cerdo y productos derivados. 


\section{MATERIAL Y METODOS}

\subsection{Muestras}

Para este estudio se recogieron muestras de canales de cerdo de diferentes mataderos de las provincias de Barcelona y Gerona. Para cada muestra se confeccionó una ficha que recogía datos del origen (Provincia de Lérida, 1 , 3 y 4 y provincia de Huesca, 2), sexo, raza (Landrace $x$ Large White), peso y categoría (E, A1, B1, C1).

Se tomó una muestra de $150 \mathrm{~g}$, aproximadamente, de músculo (carne magra del costillar) y de $100 \mathrm{~g}$ de tejido subcutáneo dorsal. Se obtuvieron así un total de 87 muestras que se conservaron a temperatura de congelación hasta el momento del análisis.

\subsection{Preparación de las muestras}

Las muestras de tejido muscular se limpiaron para eliminar al máximo los restos de grasa intermuscular, de forma que se pudiese determinar así, exclusivamente, el contenido y composición de la grasa intramuscular. Las muestras de tejido adiposo se limpiaron, igualmente, con objeto de eliminar posibles restos de tejido muscular o cartilaginoso que lo pudieran acompañar.

En todos los casos, las muestras se sometieron a trituración hasta obtener una buena homogeneización de los tejidos. Para cada determinación se tomaron dos alicuotas de cada muestra con el fin de asegurar la representatividad de los resultados.

\subsection{Métodos analíticos}

a) Extracción de la grasa: Para todas las muestras se ha utilizado el método propuesto por Folch et al. (1957).

b) Determinación de la composición en ácidos grasos por cromatografía en fase gaseosa: La preparación de los ésteres metílicos se realizó de acuerdo con el método propuesto por Slover y Lanza (1979), que utiliza $\mathrm{BF}_{3} /$ Metanol como reactivo y hexano como disolvente de extracción. El análisis cromatográfico se ha llevado a cabo en un cromatógrafo Perkin Elmer Sigma 2000, acoplado a un integrador-microordenador PE Nelson 1020 y equipado con una columna capilar tipo WCOT de sílice fundida de 50 $\mathrm{m}$ de largo y $0.25 \mathrm{~mm}$ de diámetro interno, recubierta con fase estacionaria líquida de $100 \%$ cianopropilpolisiloxano (CP Sil 88, Chrompack). Las condiciones cromatográficas fueron las siguientes: temperatura del horno isoterma a $190^{\circ} \mathrm{C}$, del inyector a $300^{\circ} \mathrm{C}$ y del detector a $270^{\circ} \mathrm{C}$. Como gas portador se utilizó Helio, a una presión de entrada de 15 psi y con una relación de split 1:100.

Para la identificación se han utilizado patrones de los diferentes ácidos grasos (Sigma). La cuantificación de los ácidos grasos se realizó por normalización interna.

\section{RESULTADOS Y DISCUSION}

En la Tabla 1, se exponen los valores medios y desviación estándar obtenidos, correspondientes a C 18:1 t (forma isomérica única en estos sustratos), total de ácidos saturados, monoinsaturados y polinsaturados en tejido adiposo y grasa intramuscular. Para el caso de ácidos trans, se obtienen unos valores análogos a los publicados por Slover et al. (1987) (grasa intramuscular) y superiores a los señalados por Lin et al. (1984). Debe destacarse que no se observan diferencias estadísticamente significativas entre los porcentajes relativos de formas trans entrè grasa intramuscular y tejido adiposo.

Tabla I

Contenidos de C 18:1 t (\% relativo) y composición lipídica simplificada de los tejidos muscular y adiposo del cerdo

\begin{tabular}{lccccc}
\hline & C18:1 t & $\%$ grasa & $\begin{array}{c}\text { Total } \\
\text { monoinsatur. }\end{array}$ & $\begin{array}{c}\text { Total } \\
\text { saturados }\end{array}$ & $\begin{array}{c}\text { Total } \\
\text { polinsatur. }\end{array}$ \\
\hline $\begin{array}{l}\text { Tejido } \\
\text { adiposo }\end{array}$ & & & & & \\
$\overline{\mathrm{x}}$ & 0.61 & & 48.9 & 36.6 & 14.5 \\
$\sigma$ & 0.53 & & 2.4 & 1.6 & 2.1 \\
max. & 1.9 & & 54.4 & 40.7 & 20.5 \\
min. & 0.1 & & 44.3 & 33.0 & 6.6 \\
$\mathrm{n}$ & 84 & & 84 & 84 & 84 \\
\hline Tejido & & & & & \\
intramuscular & & & & & \\
$\overline{\mathrm{x}}$ & 0.64 & 3.2 & 46.2 & 37.0 & 16.1 \\
$\sigma$ & 0.63 & 1.9 & 4.8 & 4.3 & 3.8 \\
max. & 4.0 & 9.5 & 61.2 & 54.7 & 27.6 \\
min. & 0 & 1.0 & 35.2 & 30.2 & 7.5 \\
$\mathrm{n}$ & 77 & 79 & 77 & 80 & 66 \\
\hline
\end{tabular}

Por otra parte, en la Tabla II, se recogen los valores medios correspondientes a los porcentajes de C 18:1 t en la grasa intramuscular, en función del sexo y origen del animal y de la categoría de la canal. El análisis de la varianza entre estos grupos revela la existencia de diferencias significativas únicamente en función del origen $(p<0.0001)$. Un contraste de hipótesis pone de manifiesto que sólo existen diferencias entre las medias de los orígenes 1 y 4 , entre 2 y 3 y entre 2 y 4 .

Tabla II

Contenidos medios de $\mathrm{C}$ 18:1 t (tejido muscular y adiposo) en los diferentes grupos de muestras establecidos en función de la categoría, el sexo y del origen

\begin{tabular}{c|ccc|ccc}
\hline & \multicolumn{3}{|c|}{ TEJIDO MUSCULAR } & \multicolumn{3}{c}{ TEJIDO ADIPOSO } \\
\hline Categoria & $\mathrm{n}$ & Media & E.E. & $\mathrm{n}$ & Media & E.E. \\
E & 5 & 0.22 & 0.27 & 5 & 0.14 & 0.21 \\
$\mathrm{~A} 1$ & 8 & 0.21 & 0.21 & 7 & 0.16 & 0.18 \\
$\mathrm{~B} 1$ & 35 & 0.89 & 0.10 & 41 & 0.83 & 0.07 \\
C1 & 29 & 0.55 & 0.11 & 31 & 0.51 & 0.09 \\
\hline Sexo & & & & & & \\
M & 15 & 0.58 & 0.99 & 14 & 0.23 & 0.08 \\
H & 48 & 0.56 & 0.55 & 54 & 0.63 & 0.61 \\
\hline Origen & & & & & & \\
1 & 14 & 0.97 & 0.14 & 16 & 0.86 & 0.05 \\
2 & 17 & 1.59 & 0.12 & 19 & 1.39 & 0.06 \\
3 & 17 & 0.61 & 0.12 & 18 & 0.27 & 0.06 \\
4 & 29 & 0.20 & 0.09 & 31 & 0.19 & 0.02 \\
\hline
\end{tabular}


Tabla III

Correlaciones entre el porcentaje de C18:1t en grasa de depósito e intramuscular y proporciones de ácidos grasos saturados, mono y polinsaturados, así como con el porcentaje de grasa intramuscular $(p a r a ~ n=55)$.

\begin{tabular}{lccccccccc}
\hline & & \multicolumn{3}{c}{ Tejido muscular } & \multicolumn{4}{c}{ Tejido adiposo } \\
& \% grasa & C18:1t & Sat & Mono & Poli & C18:1t & Sat & Mono & Poli \\
\hline C18:1t & $\mathrm{r}=0.6945$ & & 0.5464 & -0.5138 & 0.0951 & 0.7417 & 0.2080 & -0.3574 & 0.1858 \\
intramuscular & $\mathrm{p}=<0.0001$ & & $<0.0001$ & 0.0001 & 0.4897 & $<0.0001$ & 0.1276 & 0.0074 & 0.1745 \\
& & & & & & & & & \\
C18:1t & $\mathrm{r}=0.7638$ & 0.7417 & 0.4514 & 0.4579 & 0.0931 & & 0.3868 & -0.6029 & 0.2902 \\
adiposo & $\mathrm{p}=<0.0001$ & $<0.0001$ & 0.0005 & 0.0004 & 0.4992 & & 0.0035 & $<0.0001$ & 0.0316 \\
\hline
\end{tabular}

Para el caso de tejido adiposo (Tabla II) se ha aplicado el mismo tratamiento estadístico. Para los casos en que el análisis de la varianza ha sido significativo (origen, $p<$ 0.0001 y categoría, $p=0.0006$ ), se aplicó un contraste de hipótesis, resultando diferencias entre las categorfas $A 1$ y B1 y entre todos los orígenes, excepto entre 3 y 4 . La aplicación de una t de Student para varianzas no homogéneas, revela diferencias significativas entre las medias de $\mathrm{C}$ 18:1 t, en función del sexo (intervalo de confianza al $95 \%$, para la diferencia 0.57-0.23).

Finalmente,un estudio de correlación entre variables (Tabla III), revela una relación positiva entre el contenido graso intramuscular y el porcentaje de $C 18: 1 \mathrm{t}(r=0.6945$, $p<0.0001$ ), lo que permitiría concluir un posible efecto de acumulación de estas formas isoméricas. También se observa una relación positiva entre $C 18: 1$ t de la grasa intramuscular y el total de ácidos saturados $(r=0.5464, p$ $<0.0001$ ), y negativa con el total de ácidos monoinsaturados de esta fracción $(r=-0.5138, p=0.0001)$.

En relación con los porcentajes de este isómero en el tejido adiposo, se observan análogos tipos de correlación con el total de saturados $(r=0.3868, p=0.0035)$ y monoinsaturados $(r=-0.6029, p<0.0001) y$, además aunque con menor grado de significación, una correlación positiva con el total de polinsaturados $(r=0.2902, p=0.0316)$.

\section{CONCLUSIONES}

Los contenidos (\% relativos) de isómeros trans en tejido adiposo y grasa intramuscular de cerdo se sitúan, para las muestras estudiadas, dentro de valores bajos y semejantes a los señalados por distintos autores. El isómero mayoritario, prácticamente único, es la forma $\mathrm{C} 18: 1 \mathrm{t}$ no siendo cuantificables en este tipo de sustratos isómeros trans C16:1 ó C18:2.

Las diferencias significativas observadas entre animales de la misma raza procedentes de orígenes diferentes parece indicar que debe existir una influencia importante de la alimentación (Elson et al., 1981) (Royce et al., 1984). De este hecho se deduce la gran influencia que pueden tener en relación con este tema, el tipo de grasas utilizadas en la elaboración de los piensos.

Es de destacar una posible acumulación de estas formas en función del contenido graso intramuscular y también, que las correlaciones existentes entre estos conteni- dos y el total de ácidos saturados y monoinsaturados, independientemente de otras consideraciones de tipo metabólico, podrían explicarse en base a las grasas utilizadas en la elaboración de piensos y de los tratamientos a que han sido sometidas.

\section{AGRADECIMIENTOS}

Este trabajo ha sido posible gracias al disfrute de una "Ayuda a la investigación" de la Caixa de Barcelona.

\section{BIBLIOGRAFIA}

Boatella, J. (1992).- "Changes in dietary fats in the Spanish diet. Future Recommendations".- Seminar on Public Health: Changing Patterns of fat intake among Mediterranean Countries. WHO. Barcelona.

Brisson, G. (1982).- "Lipides et nutrition humaine".- Masson, Paris

British Nutrition Foundation (1987).- "Trans fatty acids".- The reports of the British Nutrition Foundation, London.

Elson, C.E., Benevenga, N.J., Canty, D.J. (1981).- "The influence of dietary unsaturated cis and trans and trans and saturated fatty acids on tissue lipids of swine".- Atherosclerosis 40, 115-137.

Emken, E.A. (1979).- "Utilization and effects of isomeric fatty acids in humans".- In "Geometrical and positional fatty acid isomers".- Ed. E.A. Emken and H.J. Dutton.- The American Oil Chemists' Soc. Champaign, Illinois.

Entressangles, B. (1986).-"Mise au point sur les isomeres trans alimentaires".- Rev. Fr. Corps Gras 33, 47-58

Folch, J., Lees, M., Sloane-Stanley, G.H. (1957).- "A simple method for isolation and purification of total lipids from animal tissues".- J. Biol. Chem. 226, 497-509.

Girard, J.P., Denoyer, C., Desmoulin, B., Gandemer, G. (1983).-"Facteurs de variation de la composition en acides gras des tissues adipeux et musculaires de porc".- Rev. Fr. Corps Gras 30, 73-79.

Girard, J.P., Denoyer, C., Bucharles, C., Gerardot, L. (1985).- "Les lipides animaux dans la filière viande (Vol. I)".- Ministere de l'Agriculture CDIUPA, Edit. Apria, Paris.

Gunstone, F.D., Norris, F.A. (1983).- "Lipids in Foods. Chemistry, Biochemistry and Technology".- Pergamon Press, Great Britain.

Hernández, N., Codony, R., Rafecas, M. Boatella, J. (1991).-"Contenidos en isómeros trans de los ácidos grasos en productos cárnicos. (I) Embutidos".- Grasas y Aceites 42, 143-147.

Hunter, J.E., Applewithe, T.H. (1986).- "Isomeric Fatty acids in the US diet: levels and health perspectives" - Am. J. Clin. Nutr. 44, 707-717.

Islam, M.N., SIhlitzer, J.L., Islam, N.B. (1983).- "Effect of trans fatty acids on protein utilization and serum cholesterol".- J. Food Sci. 48, 101-103.

Kinney, M. Cross, H.R., Smith, G.C., Smith, S.B. (1990).- "Subcellular distribution and composition of lipids in muscle and adipose tissues".- $J$ Food Sci. 55, 43-45.

Kinsella, J.E., Bruckner, G., Mai, J., Shimp, J. (1981).- "Metabolism of trans fatty acids with emphasis of the effects of trans, trans-octadecadienoate on lipid composition, essential fatty acid, and prostaglandins: an overview".- Am. J. Clin. Nutr. 34, 2307-2318. 
Kummerow, F.A. (1986).-"Dietary effects of trans fatty acids".- J. Environ. Pathol. Toxicol. 6, 123-149.

Lin, K.C., Marchello, M.J., Fisher, A.G. (1984).-- "Determination of the amount of trans-octadecenoate and trans-9, trans-12-octadecenoate in fresh lean and fatty tissues of pork and beef".- J. Food Sci. 49, 15211524.

Royce, S.M., Holmes, R.P.,Takagi, T., Kummerow, F.A. (1984).- "The influence of dietary isomeric and saturated fatty acids on atherosclerosis and eicosanoid synthesis in swine".- Am J. Cllin. Nutr. 39, 215-222.

Slover, H.T., Lanza, E. (1979).- "Quantitative analysis of food fatty acids by capillary gas chromatography".- J. Am. Oil Chemists' Soc. 56, 933-943.

Slover, H.T., Thomson, R.H., Davis, C.S., Merola, G.V. (1987).- "The lipid composition of raw and cooked fresh pork".- J. Food Comp. Analysis 1, 38-52.

Sommerfeld, M. (1983).--Trans unsaturated fatty acids in natural products and processed foods".- Prog. Lipids Res. 22, 221-233.

Zevenbergen, J.L. (1987).-“Biological effects of trans fatty acids". In "Fat production and consumption".- Ed. by Galli and Fedelli.- NATO/Plenum Press, New York.

(Recibido: Febrero 1993) 